\title{
Highly Manoeuvrable Eversion Robot Based on Fusion of Function with Structure
}

\author{
T. Abrar*1, Student Member, IEEE, F. Putzu*1, Student Member, IEEE, A. Ataka ${ }^{2}$, \\ H. Godaba ${ }^{1}$ and K. Althoefer ${ }^{1}$, Senior Member, IEEE
}

\begin{abstract}
Despite their soft and compliant bodies, most of today's soft robots have limitations when it comes to elongation or extension of their main structure. In contrast to this, a new type of soft robot called the eversion robot can grow longitudinally, exploiting the principle of eversion. Eversion robots can squeeze through narrow openings, giving the possibility to access places that are inaccessible by conventional robots. The main drawback of these types of robots is their limited bending capability due to the tendency to move along a straight line. In this paper, we propose a novel way to fuse bending actuation with the robot's structure. We devise an eversion robot whose body forms both the central chamber that acts as the backbone as well as the actuators that cause bending and manoeuvre the manipulator. The proposed technique shows a significantly improved bending capability compared to externally attaching actuators to an eversion robot showing a $133 \%$ improvement in bending angle. Due to the increased manoeuvrability, the proposed solution is a step towards the employment of eversion robots in remote and difficult-to-access environments.
\end{abstract}

\section{INTRODUCTION}

Soft robots are lightweight, compliant, and low cost compared to their rigid counterparts. Moreover, they are easy to transport and deploy. Thanks to their inherent compliance, they can passively interact with the surrounding environment, such as de-shaping or bending when colliding with an obstacle. To manoeuvre soft continuum manipulators, researchers have developed and implemented different actuation solutions; such as tendon mechanisms [1], [2], McKibben muscles [3], pouch motors [4], [5], inflatable pleats [6], inflatable bladders [7], [8], inverse pneumatic artificial muscles [9] and antagonistic pneumatic artificial muscles [10]. Despite these advancements, large scale applications of soft manipulators are hindered by their limited elongation capability.

One particular class of inflatable robots based on the eversion principle has been shown to overcome this issue and is capable of growing longitudinally from a very small size to an enormous length achieving extension ratios of 1 (initial length) to 100 (fully extended) [11]. These robots provide practical solutions for the exploration of otherwise inaccessible, remote environments [12]

*Equally contributing authors;

${ }^{1}$ T. Abrar, F. Putzu, , H. Godaba, K. Althoefer are with the Centre for Advanced Robotics@ Queen Mary (ARQ), Queen Mary University of London, Mile End Road, London, E1 4NS, United Kingdom (phone: +44 (0)20 7882 3419; e-mail:\{t.abrar, f.putzu, h.godaba, k.althoefer\}@qmul.ac.uk).

${ }^{2} \mathrm{~A}$. Ataka is with Department of Electrical Engineering and Information Technology, Gadjah Mada University, Indonesia (phone: +62 274 588688; email: ahmad.ataka.ar@mail.ugm.ac.id).

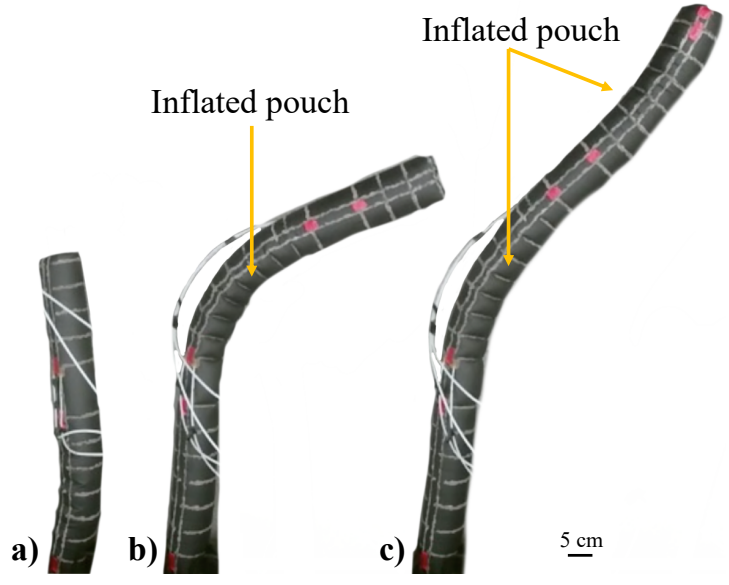

Figure 1. The prototype of an eversion robot with an integrated actuation system. a) Initial folded state, b) intermediate state, with 1 active pouch, c) extended state with 2 active pouches.

Structurally, the eversion robot is like a cylindrical sleeve which is folded inwards [13]. When pressurized the folded part turns inside out, resulting in longitudinal displacement or growth at the robot tip. The robot tends to extend along a straight line in free space. Because of their soft structure and is capable of conforming to their surroundings, these robots are suitable to move in unstructured environments. Research efforts have focussed on exploiting these advantages, and eversion robots have been proposed for applications in various areas, including minimally invasive surgery [14], [15] search and rescue, the inspection of inaccessible places [16] [19], telecommunication [20] and artificial muscle [21].

The capability to manoeuvre in the lateral direction is an important capability for a robot arm enabling the exploration of surrounding environments as well as following trajectories to conduct required tasks, such as manipulation of objects or the delivery of sensors. Various techniques to bend and navigate continuum manipulators have been implemented and researched. Previously, continuum manipulators were bent by creating tension through tendons attached to their body [22], [23]. Another method to create bending in soft continuum manipulators is by using pneumatic/hydraulic actuation [24], [25], shape memory alloys [26], or McKibben muscles [27], [28]. However, the unfolding process that the tip of the eversion robot experiences causes a high bending strain considerably limiting the types of actuators that can be integrated. Only actuators that can be compactly incorporated in the ro- 
bot sleeve and that can adapt to the unfolding process can be considered. Greer et al. proposed to navigate eversion robots in unstructured environments exploiting the robot's interaction with fixed obstacles [29]. However, navigation cannot be achieved in free space and active manoeuvring is not possible using this technique. Hawkes et al. used multiple series of latches along the longitudinal side of the robot which open based on the pressure applied to the chamber with latches [11]. The drawback of this approach is that the bending is not reversible, as once a set of latches have opened, the robot is in a configuration that is fixed from then on. Another approach uses three sets of pneumatic artificial muscles externally attached to the surface of an eversion robot along its whole length [17], [30], [31]. The advantages of this solution are the reversibility of the bending and the active control over the system. However, in these works, the actuators were attached to the outside of the main robot body.

In this paper, we develop a novel method of integrating bending actuation into the body of the eversion robot. In contrast to existing methods of augmenting a robotic backbone with different types of actuators, we develop an eversion robot whose body itself forms the pneumatic pouch actuators that cause bending and manoeuvre the manipulator. The robot can be elongated longitudinally by pressurizing the main chamber and actuating a given set of pouches in the periphery enables it to bend sidewards (as shown in Figure 1). The direct fusion of the bending actuators and the central chamber from the same two-layered fabric construction allows a change in the robot's cross-section improving its bending capability. Through this approach, the bending capability of the eversion robot is improved by up to $133 \%$ compared to the existing technique of attaching actuation pouches externally. Also, the robot is easier to fabricate and highly robust owing to the multipurpose structure that functions as both the backbone and the actuation mechanism. This work makes the following important contributions:

1) a novel methodology for fusing the wall of the robot body and bending mechanism, and

2) achievement of a highly manoeuvrable inflatable robot arm with bending improved by over $100 \%$.

The paper is organized as follows. Section II outlines the working principle of the integrated pouches. In Section III, the fabrication of the eversion robot with integrated pouches is presented. Section IV describes the analytical model for simulating bending. Section V presents the analytical and experimental results comparing our proposed methodology with the existing technique and Section VI presents conclusions and future directions.

\section{WORKING PRINCIPLE}

The proposed eversion robot with pneumatic pouches integrated into its peripheral has two mechanisms of actuation longitudinal elongation, and lateral bending. Longitudinal elongation of the robot arm takes place when air pressure is applied to the central chamber encompassed by the fabric causing material to evert from the tip thereby extending in length. The second is the bending actuation which allows the robot to bend laterally when the pressure is applied to the pouches (Figure 2).
These two mechanisms can be independently controlled. Analogous to a traditional manipulator, the central chamber can be pressurized first until the robot has everted to its maximum length and the pouches can be pressurized to create bending in different segments of the robot. Alternatively, it is also possible to actuate pouches to bend and change direction while everting. This feature is important if one wants to advance into a remote environment and navigate simultaneously. The pressurized central chamber running along the entire length of the robot acts as a backbone. Each segment of the eversion robot has two sets of pouches located on either side of the main body which can be actuated using two dedicated pneumatic lines. When one set of pouches are actuated by applying pressure, the pouches inflate contracting laterally. Due to the differential shortening of their length on one side of the robot body, it bends towards the direction of the actuated pouch. (Figure 2(c)).

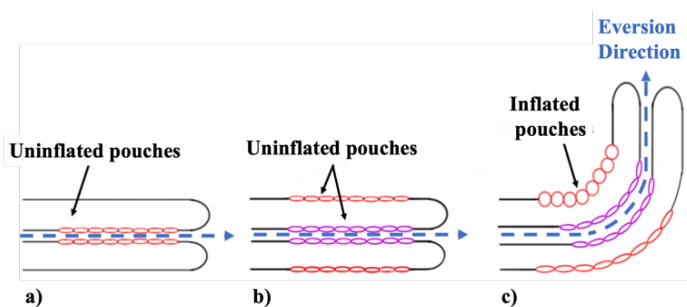

Figure 2. Eversion and bending principle of the robot; (a) initially when the robot starts growing the pouches are folded inside (b) when the previously folded part everts the pouches come outside (c) by inflating the pouches on one side of the robot the bending occurs.

The more the pouches are inflated, the more the robot will bend [32], [33]. The pressure equilibrium, between pouches and the main chamber, also influences the bending performance of the robot. The lower the pressure in the main chamber and the higher the pressure in the pouches, the greater is the bending angle that the robot achieves. On the other hand, the higher the pressure in the main chamber, the stiffer the robot body gets and becomes more resistant to bending.
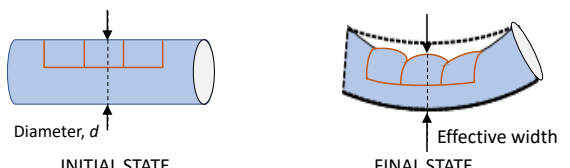

(a) Side View

FINAL STATE

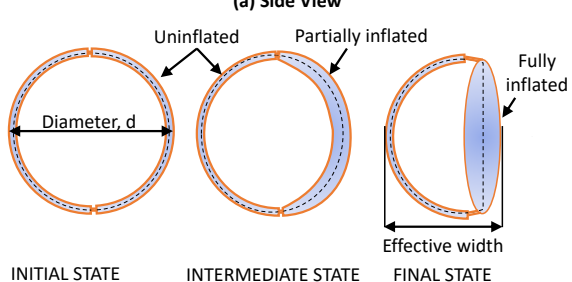

(b) Cross-section View

Figure 3. (a) Illustration of the side view of the eversion robot before and after a segment of pouches are actuated. The actuation of pouches causes contraction along the longitudinal direction and constriction in the circumferential direction. (b) The cross-sectional view of the eversion robot at different levels of pouch inflation. As the pouch inflates to higher pressures, the effective width decreases. 
In addition to the contraction, inflating the pouch actuators that are tightly fused with the robot structure also leads to a dramatic change in the cross-section of the robot's main chamber Figure 3(a). In the initial state with only the central chamber pressurized, the central chamber has a circular cross-section Figure 3(b). Applying pressure to a pouch not only causes longitudinal contraction but also creates a change in its causes it to contract along the circumferential direction. In consequence, the cross-section deviates from its initial circular shape and creates a constriction in the robot arm (as shown in Figure 3. This reduction in the effective width plays a major role in enhancing the bending capability of the eversion robot, as will be discussed in further sections.

\section{FABRICATION}

To construct an eversion robot with a fused bending system, the sets of pouches are constructed first. A set of pouch actuators is created by sewing two overlapping fabric sheets (coated microfibre polyester, RipStop ${ }^{\mathrm{TM}}$ ) together (Figure 4a). The 2D pattern (shown in Figure 4b) is then sewn into the double-layered fabric sheet employing a 'heavy-duty' sewing machine $\left(\right.$ Singer $\left.{ }^{\mathrm{TM}}\right)$. The seams are then made airtight by applying a thin layer of 'Bentley' dipping latex.

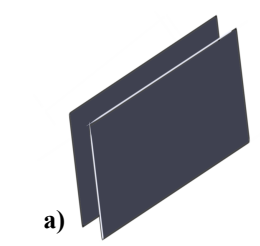

b)
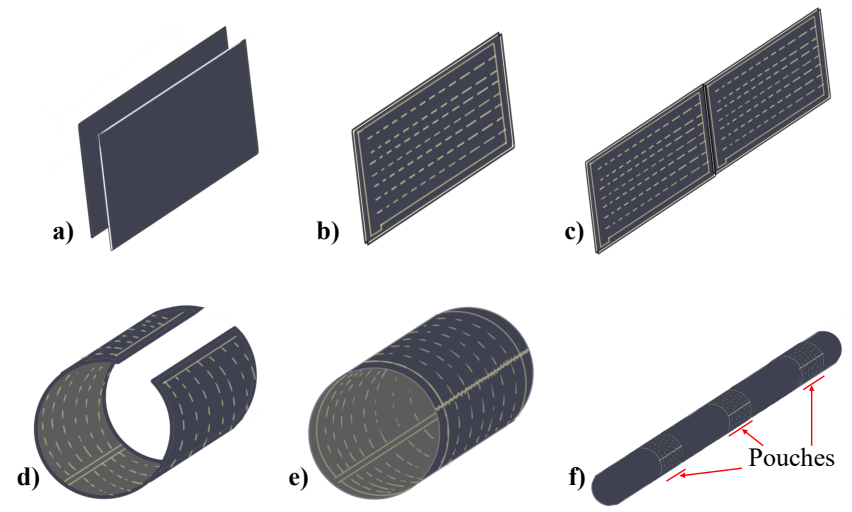

Figure 4. Schematic of the fabrication process. a) two fabric sheets overlapped, b) pattern sewn into the fabric, c) two sets of pouches joined together, d) pouches folded in half, e) two halves are sewn together to create the 1-DoF joint, f) complete robot with 3 integrated joints.

A similar procedure is applied to create another set of pouches alongside the first one. This pair of pouches created is then folded (Figure 4d) and their free edges are sewn together to form a cylindrical structure (Figure 4e). One of the two ends of the cylinder is closed while the other end is kept open for the air inlet, and all the seams are sealed with latex. The tubular structure is then turned inside out partially (Figure 4f). When the central chamber is pressurized, the structure gradually everts from the tip, generating longitudinal translation of the eversion robot.

When a set of pouches is pressurized, the flat sheets of fabric (Figure 5a) bulge outward (Figure 5b), causing the shortening in length. Considering that the fabric is inextensible, the maximum linear strain that can theoretically be achieved by the contraction of the pouches is $36.34 \%$. This corresponds to the pouches assuming a cylindrical shape. Practically, the achievable longitudinal strain is guided by several factors such as the flexural rigidity of the material of the pouches, the geometrical dimensions of the length and the width, and the type of seal of the pouch edges.

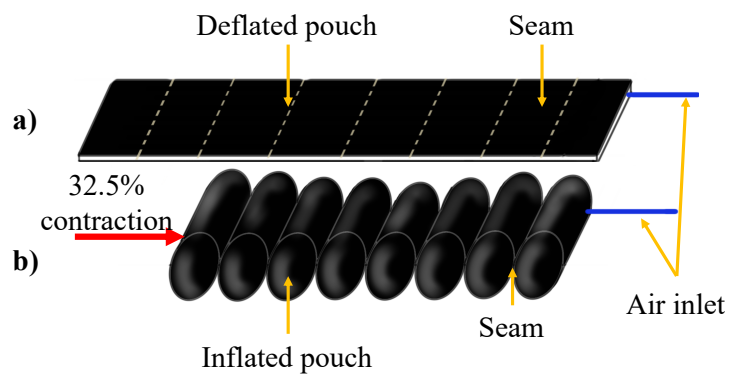

Figure 5. Design and working principle of the actuator. a) Pouch in a deflated flat state, b) inflated contracted pouch.

As the actuation strain of the pouches is crucial to the bending capability, we experimentally test the fabric pouches (in Figure 5(b)) created using our technique. A set of 8 pouches, each with a dimension of $5 \mathrm{~cm} \times 13 \mathrm{~cm}$ with a single pressure inlet are inflated until maximum contraction. When the pouches are in a relaxed state, their initial length $l_{0}$ is 0.4 $\mathrm{m}$. When inflated, the length becomes $l_{\min }=0.27 \mathrm{~m}$. This results in a maximum strain (as shown in Figure 5) of:

$$
\varepsilon_{\max }=\Delta l / l_{o}=\left(l_{o}-l_{\text {min }}\right) / l_{o}=32.5 \%
$$

This value is comparable to the maximum theoretical limit, which is $36.34 \%$ [34] and is a marked improvement compared to similar techniques in the literature such as the Pouch Motor actuator [5] which contracts $28 \%$ of its original length and the HASEL actuator [34] with a maximum stroke of $18 \%$ compared to its original length. This increase may be attributed to two reasons: (i) the high flexibility of the fabric, and (ii) the seams in the fabric are thinner compared to seams made by sealing two plastic sheets. This strain capability is linked to the bending capability which will be discussed in the next section.

\section{Analytical Modelling}

In this section, we develop a theoretical model to estimate the bending in the proposed eversion robot with pouches fused into the structure. We model the eversion robot as an inflated beam whose structure is composed of the fused pouches [35]. In effect, a pressure $P_{m}$ applied to the main chamber of nominal radius, $\mathrm{R}$ generates an axial force, $F_{a x}=P_{m} \pi R^{2}$ acting on the membrane composing the pouches, in the longitudinal direction (Figure 6a).

Due to the symmetry of the pouches located on either side of the central robot axis, one segment of pouches experiences tension equal to half of the total axial force. Consequently, the force acting longitudinally on a set of pouches is given by:

$$
F_{p}=F_{a x} / 2=P_{m} \pi R^{2} / 2
$$

At the same time, the pressure applied to a set of pouches causes the pouches to inflate and thereby contract axially. Let us consider the membrane of a single pouch of initial length, $l_{o}$. Upon application of pressure $P_{p}$, it deforms into an arc of a circle subtending an angle $\theta$ at the centre (Figure $6 b)$. Assuming that the pouch retains this uniform shape of 
the curve across its width, $D$, the volume of air enclosed by the pouch as a function of the subtended angle is given by [5]:

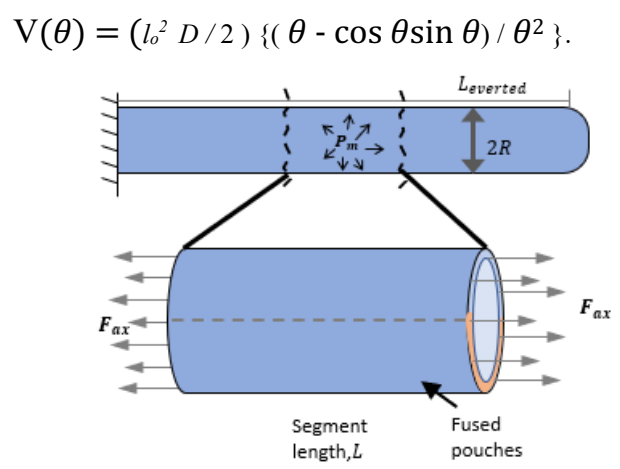

(a) Forces in the eversion robot

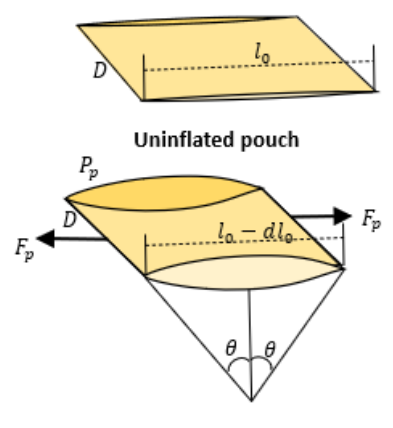

Inflated pouch

(b) Free body diagram of inflated pouch

(c) Geometry of bending
Figure 6. Bending segment with constant curvature. (a) Forces in the eversion robot, (b) free body diagram of inflated and uninflated pouch, (c) geometry of bending.

According to the conservation of energy, the work done by the air pressure in inflating the membrane is equal to the energy due to the virtual translation of longitudinal force $F_{p}$, giving:

$$
-F_{p} d l_{o}=P_{p} d V
$$

From equation (3) and equation (4), we get,

$$
F_{p}(\theta)=l_{o} D P_{p}(\cos \theta) / \theta .
$$

From geometry, the linear pouch contraction ratio, $\mathcal{E}$, is:

$$
\varepsilon=1-\{(\cos \theta) / \theta\}
$$

For a given pouch pressure $P_{p}$ and the main chamber pressure $P_{m}$, the axial force generated through the main chamber pressure and the contraction force due to pouch inflation are in equilibrium. We obtain from equations (2) and (5):

$$
F_{p}(\theta)=F_{m} / 2 .
$$

Substituting the relations for $F_{p}$ and $F_{m}$, we get

$$
l_{o} D P_{p}(\cos \theta) / \theta=P_{m} \pi R^{2} / 2
$$

For a given combination of pouch pressure and main chamber pressure, we solve equation (8) to obtain the angle of the membrane bending, $\theta$, and the strain in the set of pouches is then calculated from equation (6).

When one segment of length, $L$ composed of a set of pouches is actuated with pressure $P_{p}$, the pouches contract by a factor, $\varepsilon$, while the unactuated segment retains its original length, $L$. A geometric model of the resultant bending in a segment is shown in Figure 6c. For a beam of constant width given by $d$, the total bending angle of the segment is given by

$$
\theta_{\text {const }}=\varepsilon L / D \text {. }
$$

However, for a beam with a non-uniform width given by $d(l)$, the beam undergoes inhomogeneous bending (Figure $6 \mathrm{c})$. The total bending angle of a segment of length $L$ is:

$$
\theta_{v a r}=\int(\varepsilon / d(l)) d l
$$

where the limit of the integral extends from 0 to $L$.

Since $\varepsilon$ has a maximum limit, the maximum bending angle that can be achieved attaching a pouch actuator externally to the walls of an eversion robot is given by the diameter and the length as we can see from the equation (9). However, this is not the case for our proposed eversion robot. Integrating the pouches into the body (i.e., the outer fabric layer) of the eversion robot results in a phenomenon whereby the two opposite pouches get closer to each other during the inflation of one of the pouches. As distance $d$ becomes smaller, equation (10) shows that the maximum bending angle $\theta_{\text {const }}$ will be greater than $\theta_{\text {var }}$. Hence, it can be inferred that the proposed solution has the potential to create a larger bending angle than the limit expressed in equation (10), improving the overall bending capability of the eversion robot. Analytical results and experimental studies to evaluate this hypothesis are shown in the next section.

\section{RESUlts}

\section{A. Experimental Setup}

This experiment focuses on the evaluation of the bending capability of the proposed eversion robot. Each bending segment is $40 \mathrm{~cm}$ long and has the same diameter as the eversion robot. The robot has 3 bending segments and 2 connecting links in between two segments, each $10 \mathrm{~cm}$ long. Without loss of generality, we only test the bending of the middle segment. The experimental setup is depicted below in Figure 7.
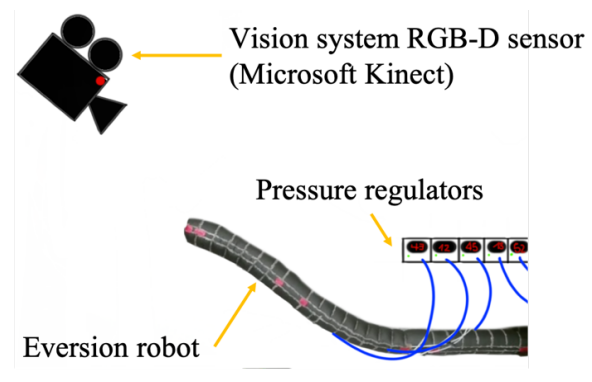

Figure 7. The experimental setup for our eversion robot. 
To pressurise the main chamber of the robot and the pouches, electronic proportional valves (SMC ITV 2050212BL4) are employed. An RGB-D sensor (Microsoft Kinect) is ceiling mounted above the robot to record the image of the robot's configuration during each experimental step. The camera is used to detect the change in the diameter of the robot's main body at several points along the bending segment. Additionally, to measure the bending angle, a pair of red markers are attached to the robot body on either ends of the bending segment. The angle between the two lines passing through these two pairs of markers characterises the bending angle.

We perform 2 main experiments to prove our hypothesis. The first experiment shows that this fusion leads to a change in the diameter of the robot when pouches are actuated. The second does a comparative evaluation of bending in an eversion robot with fused pouches with that with externally attached pouches. Finally, an analytical model based on Section IV is used to validate this hypothesis.

\section{B. Morphing of the cross-section to increase bending ac- tuation}

We evaluate our hypothesis that the integration of the actuator inside the robot's structure will bring a change in the cross-section of the robot's main chamber. For this purpose, we exploit the images retrieved from the RGB-D sensor to measure the effective width (denoted in Figure $8 \mathrm{a}$ ) of the robot in three chosen locations along the bending segment. Moreover, we also detect the diameter of the robot at two additional locations on either end of the bending segment. The five locations where we measured the robot diameters are shown in Figure 8. The pressure in the main chamber is kept constant at 0.3 bar. The pneumatic pressure in the pouches is increased in steps from 0 to 2.5 bar with an increment of 0.5 bar at each step.

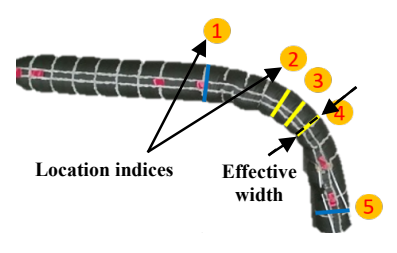

(a)

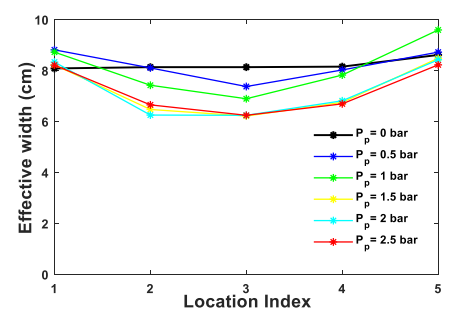

(b)
Figure 8. (a) Image of the eversion robot showing constriction in the width of the main chamber. Locations 1-5 refer to regions on the eversion robot that are monitored to study the variation in cross-section. (b) The plot of the effective width measured at the 5 different locations for various values of pressure in the pouches when the main chamber pressure is $0.3 \mathrm{bar}$.

As the pouch pressure is increased, the effective width of the robot arm in the central regions of the bending section denoted by Location Indices 2,3 and 4 decreases significantly. The distance between two pouches at locations 1 and 5 do not change considerably when different pressure values are given to the robot. Compared to the initial width at zero pouch pressure, we note a change of $18 \%, 23 \%$, and $18 \%$, respectively at locations denoted by 2,3 and 4 (Figure $8 \mathrm{~b}$ ).
Further, we see that the change in the width saturates around a pouch pressure of 1.5 bar and does not decrease upon the further increase in the pressure indicating that the pouches may have reached the state shown in Figure 3b. However, the pouches may further undergo longitudinal contraction when pressure is increased beyond 1.5 bar.

\section{Experimental comparison with the externally attached pouches}

We then conduct experiments to analyse the difference between our proposed fused actuation structure and an eversion robot where pouch actuators are attached externally (Figure 9a). The pressure in the main chamber is regulated at 0.3 bar while the pressure in the pouches is increased from 0 to 4 bar in steps of 0.5 bar as shown in Figure 9. We first test the eversion robot with the fused pouches (Figure 9b) and then externally attach another set of fabricated pouches and evaluate the bending characteristics by actuating the external set of pouches (Figure 9c).
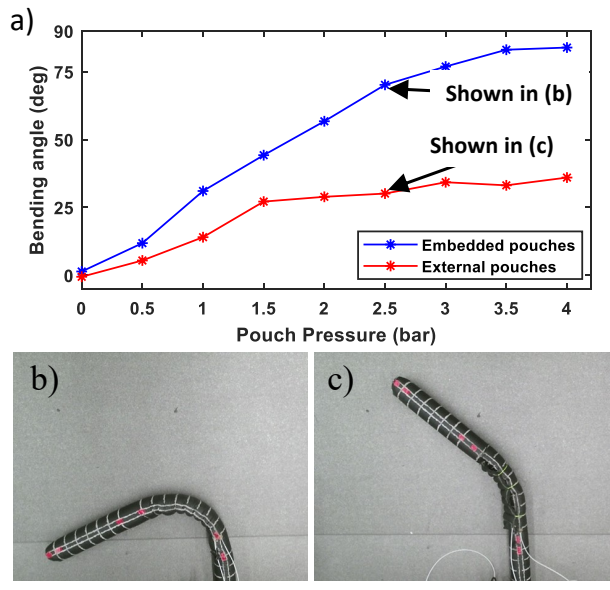

Figure 9. Bending comparison (a)The plot of bending angles as a function of pressure in the pouches for various pressure values applied to the main chamber for the case of integrated pouches (solid lines) and external pouches (dash lines). The pose of the eversion robot with pressure in the main chamber ( 0.3 bar) and in the pouches $(2.5$ bar) for the case of (b) integrated pouches and (c) external pouches.

The bending angle in both the prototypes increases with an increase in pouch pressure. The bending angle produced by an eversion robot with integrated pouches is $83.94^{\circ}$ while the maximum bending angle produced in a robot with externally attached pouches is $36.03^{\circ}$. This corresponds to a $133 \%$ improvement for the bending angle. These results show that the fused pouches can achieve significantly higher bending compared to externally attached pouch actuators.

\section{Analytical results}

We now utilise the analytical model developed in Section IV to simulate the bending in an eversion robot with external and embedded pouches. For this simulation, we consider a pouch segment of total length $40 \mathrm{~cm}$ and each pouch of length $5 \mathrm{~cm}$ and width $13 \mathrm{~cm}$. The initial radius of the robot arm before the actuation of the pouches is $4.5 \mathrm{~cm}$. These dimensions are consistent with the experimental prototypes tested above. The key difference between internal and externally attached pouches, in terms of modelling is the diameter change caused due to the fused pouch structure. Such a diam- 
eter change is absent in the prototype with pouches attached externally. So, bending caused by the longitudinal strain of the pouches is evaluated using equations (9) and (10) for external and embedded(fused) pouches respectively.

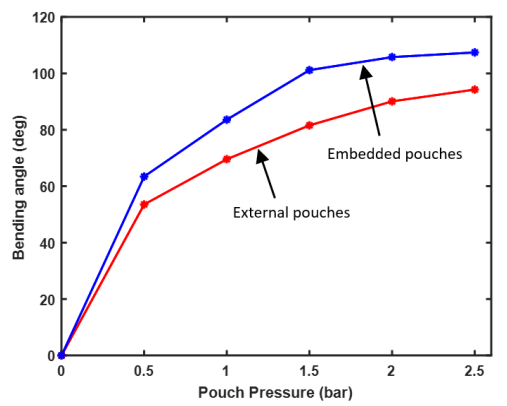

Figure 10. Comparison of simulated bending angles at different applied pouch pressures for eversion robots with embedded pouches (shown in blue) and externally attached pouches (shown in red). The main chamber's pressure is 0.3 bar.

As it is complex to directly model the diameter change caused by inflation of the pouches in the fused structure, we utilise experimentally obtained values for changes in effective width shown in Figure 8. The simulation results (Figure 10) show that the bending angle in the robot with fused pouches is higher than that in the robot with external pouches. This confirms out the hypothesis that the change of effective diameter (caused by using the actuation pouches integrated with the outer layer of the main body of the robot) enables the robot to achieve higher bending angles. However, the difference in bending angles between the fused and externally attached pouches is much higher in the experiments as compared to those predicted by the theoretical model. This difference between experiment and theory is due to the following reasons. While the pouches are assumed to be at the periphery of the robot body in both cases, the externally attached pouches are further away from the centre than the fused pouches which is not accounted for in the model. The effective width measured from the images which are used in the analytical calculation of the bending angle for the fused pouches may be larger than the actual width due to the angle of the camera.

Furthermore, we note that the analytical results overestimate the bending angles compared to the experimentally recorded values. This is because the model considers the pouches to inflate like ideal cylinders. However, in the actual scenario, the edges of the pouches are sealed and cannot completely morph into cylinders with a circular cross-section throughout, as shown in Figure 5. Due to these, the volume of the real inflated pouch is below that of the pouch estimated using the model, resulting in the slight overestimation of the bending angle in the model. The model also excludes factors such as bending rigidity of the robot parts including fabrics and pneumatic tubes and the friction forces between the robot arm and the ground.

\section{E. Maximum Bending capability}

The bending achieved due to the actuation of pouches also depends upon the pressure of the central chamber. As the pressure in the central chamber increases, the robot arm's stiffness increases, and vice-versa. To check the maximum bending capability of our robot, we depressurize the main chamber to 0.04 bar and actuate the pouches on one side to 1.4 bar. The experimental image of the eversion robot is achieving a high bending angle of $146^{\circ}$ is shown in Figure 11. At this main chamber pressure, the stiffness and the flexural rigidity of the robot are very low. Therefore, this extreme bending ability of the robot can be used in scenarios where the robot needs to make very sharp bends.

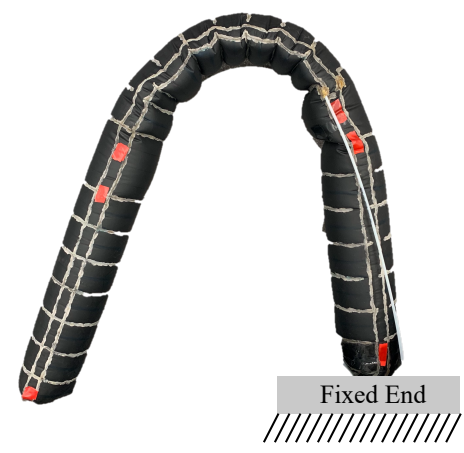

Figure 11. Eversion robot performing a $146^{\circ}$ rotation, at 0.04 bar pressure in the main chamber.

It also confirms our hypothesis that fusing the actuation into the structure, improved performance in bending capability, which positively impacts on the manoeuvrability of the robot. Having this manoeuvring ability, the proposed eversion robot is a promising candidate to be employed in unstructured environments with tight spaces or narrow openings to manoeuvre and cluttered with obstacles.

\section{CONCLUSIONS}

In this paper, we presented a novel method of integrating bending actuation into the body of the eversion robot. This integration shows promising results in terms of the robot's bending capability when compared to eversion robots equipped with externally attached pouches. Our robot was able to bend $83.94^{\circ}$ which is $133 \%$ more than the bending achievable by an eversion robot of similar dimensions equipped with external pouches at the same pressure inputs. The higher bending angles achievable through our proposed technique are attributed to change in cross-section of the main chamber occurring during bending actuation. This mechanism is discussed through experiments and simulations. The ease of fabrication, compactness of the robot structure and the robustness offered due to the fusion of actuation into the structure make this eversion robot amenable for practical applications in the field. Further, this work paves the way for the holistic design of soft robots in which different functionalities such as manoeuvring, and stiffness control are fused into a single structure. In our future work, we will address challenges in modelling and control of the robot arising from continuously changing morphology.

\section{ACKNOWLEDGMENT}

This work was supported by the EPSRC National Centre for Nuclear Robotics project (EP/R02572X/1) and the Innovate UK WormBot project (104059). 


\section{REFERENCES}

[1] K. Althoefer, "Antagonistic actuation and stiffness control in soft inflatable robots," Nat. Rev. Mater., vol. 3, no. 6, pp. 76-77, Jun. 2018, doi: 10.1038/s41578-018-0004-0.

[2] A. Shiva et al., "Tendon-Based Stiffening for a Pneumatically Actuated Soft Manipulator," IEEE Robot. Autom. Lett., vol. 1, no. 2, pp. 632-637, Jul. 2016, doi: 10.1109/LRA.2016.2523120.

[3] H. Al-Fahaam, S. Davis, and S. Nefti-Meziani, "The design and mathematical modelling of novel extensor bending pneumatic artificial muscles (EBPAMs) for soft exoskeletons," Robot. Auton. Syst., vol. 99, pp. 63-74, Jan. 2018, doi: 10.1016/j.robot.2017.10.010.

[4] S.-Y. Chang et al., "Design of small-size pouch motors for rat gait rehabilitation device," in 2015 37th Annual International Conference of the IEEE Engineering in Medicine and Biology Society (EMBC), Milan, Aug. 2015, pp. 4578-4581, doi: 10.1109/EMBC.2015.7319413.

[5] R. Niiyama, X. Sun, C. Sung, B. An, D. Rus, and S. Kim, "Pouch Motors: Printable Soft Actuators Integrated with Computational Design," Soft Robot., vol. 2, no. 2, pp. 59-70, Jun. 2015, doi: $10.1089 /$ soro.2014.0023.

[6] S. Voisembert, N. Mechbal, A. Riwan, and A. Aoussat, "Design of a Novel Long-Range Inflatable Robotic Arm: Manufacturing and Numerical Evaluation of the Joints and Actuation," J. Mech. Robot., vol. 5, no. 4, p. 045001, Oct. 2013, doi: 10.1115/1.4025025.

[7] J. Realmuto and T. Sanger, "A robotic forearm orthosis using soft fabric-based helical actuators," in 2019 2nd IEEE International Conference on Soft Robotics (RoboSoft), Seoul, Korea (South), Apr. 2019, pp. 591596, doi: 10.1109/ROBOSOFT.2019.8722759.

[8] M. T. Gillespie, C. M. Best, and M. D. Killpack, "Simultaneous position and stiffness control for an inflatable soft robot," May 2016, pp. 1095-1101, doi: 10.1109/ICRA.2016.7487240.

[9] E. W. Hawkes, D. L. Christensen, and A. M. Okamura, "Design and implementation of a 300\% strain soft artificial muscle," May 2016, pp. 4022-4029, doi: 10.1109/ICRA.2016.7487592.

[10] N. Usevitch, A. M. Okamura, and E. W. Hawkes, "APAM: Antagonistic Pneumatic Artificial Muscle," p. 8.

[11] E. W. Hawkes, L. H. Blumenschein, J. D. Greer, and A. M. Okamura, "A soft robot that navigates its environment through growth," Sci. Robot., vol. 2, no. 8, p. eaan3028, Jul. 2017, doi: 10.1126/scirobotics.aan3028.

[12] J. D. Greer, L. H. Blumenschein, R. Alterovitz, E. W. Hawkes, and A. M. Okamura, "Robust Navigation of a Soft Growing Robot by Exploiting Contact with the Environment," ArXiv190808645 Cs, Aug. 2019, Accessed: Sep. 02, 2019. [Online]. Available: http://arxiv.org/abs/1908.08645.

[13] F. Putzu, T. Abrar, and K. Althoefer, "Plant-Inspired Soft Pneumatic Eversion Robot," in 2018 th IEEE In- ternational Conference on Biomedical Robotics and Biomechatronics (Biorob), Enschede, Netherlands, Aug. 2018, pp. 1327-1332, doi: 10.1109/BIOROB.2018.8487848.

[14] S. M. Hadi Sadati, Y. Noh, S. Elnaz Naghibi, A. Kaspar, and T. Nanayakkara, "Stiffness Control of Soft Robotic Manipulator for Minimally Invasive Surgery (MIS) Using Scale Jamming," in Intelligent Robotics and Applications, vol. 9246, Cham: Springer International Publishing, 2015, pp. 141-151.

[15] F. Putzu, T. Abrar, J. Konstantinova, and K. Althoefer, "Minimally Invasive Surgery Eversion Overtube," Genoa, Italy, Mar. 2019, vol. 9th, p. 2.

[16] K. Miyasaka, G. Kawano, and H. Tsukagoshi, "Longmover: Flexible Tube In-pipe Inspection Robot for Long Distance and Complex Piping," in 2018 IEEE/ASME International Conference on Advanced Intelligent Mechatronics (AIM), Auckland, New Zealand, Jul. 2018, pp. 1075-1080, doi: 10.1109/AIM.2018.8452690.

[17] M. M. Coad et al., "Vine Robots: Design, Teleoperation, and Deployment for Navigation and Exploration," ArXiv190300069 Cs, Feb. 2019, Accessed: Apr. 03, 2019. [Online]. Available: http://arxiv.org/abs/1903.00069.

[18] Y. Ozkan-Aydin et al., "Nutation Aids Heterogeneous Substrate Exploration in a Robophysical Root," in 2019 2nd IEEE International Conference on Soft Robotics (RoboSoft), Seoul, Korea (South), Apr. 2019, pp. 172-177, doi: 10.1109/ROBOSOFT.2019.8722717.

[19] J. Luong et al., "Eversion and Retraction of a Soft Robot Towards the Exploration of Coral Reefs," in 2019 2nd IEEE International Conference on Soft Robotics (RoboSoft), Seoul, Korea (South), Apr. 2019, pp. 801-807, doi: 10.1109/ROBOSOFT.2019.8722730.

[20] L. H. Blumenschein, L. T. Gan, J. A. Fan, A. M. Okamura, and E. W. Hawkes, "A Tip-Extending Soft Robot Enables Reconfigurable and Deployable Antennas," IEEE Robot. Autom. Lett., vol. 3, no. 2, pp. 949-956, Apr. 2018, doi: 10.1109/LRA.2018.2793303.

[21] T. Abrar, F. Putzu, J. Konstantinova, and K. Althoefer, "EPAM: Eversive Pneumatic Artificial Muscle," in 2019 IEEE International Conference on Soft Robotics (RoboSoft), Seoul, Apr. 2019, p. 6.

[22] F. Maghooa, A. Stilli, Y. Noh, K. Althoefer, and H. A. Wurdemann, "Tendon and pressure actuation for a bio-inspired manipulator based on an antagonistic principle," May 2015, pp. 2556-2561, doi: 10.1109/ICRA.2015.7139542.

[23] A. Stilli, H. A. Wurdemann, and K. Althoefer, "Shrinkable, stiffness-controllable soft manipulator based on a bio-inspired antagonistic actuation principle," Sep. 2014, pp. 2476-2481, doi: 10.1109/IROS.2014.6942899.

[24] K.-H. Lee et al., "Nonparametric Online Learning Control for Soft Continuum Robot: An Enabling 
Technique for Effective Endoscopic Navigation," Soft Robot., vol. 4, no. 4, pp. 324-337, Dec. 2017, doi: 10.1089/soro.2016.0065.

[25] A. Arezzo et al., "Total mesorectal excision using a soft and flexible robotic arm: a feasibility study in cadaver models," Surg. Endosc., vol. 31, no. 1, pp. 264273, Jan. 2017, doi: 10.1007/s00464-016-4967-x.

[26] C. Laschi, M. Cianchetti, B. Mazzolai, L. Margheri, M. Follador, and P. Dario, "Soft Robot Arm Inspired by the Octopus," Adv. Robot., vol. 26, no. 7, pp. 709727, Jan. 2012, doi: 10.1163/156855312X626343.

[27] T. G. Thuruthel, M. Manti, E. Falotico, M. Cianchetti, and C. Laschi, "Induced Vibrations of Soft Robotic Manipulators for Controller Design and Stiffness Estimation," p. 6.

[28] H. B. Gilbert and I. S. Godage, "Validation of an Extensible Rod Model for Soft continuum Manipulators," in 2019 2nd IEEE International Conference on Soft Robotics (RoboSoft), Seoul, Korea (South), Apr. 2019, pp. 711-716, doi: 10.1109/ROBOSOFT.2019.8722721.

[29] J. D. Greer, L. H. Blumenschein, A. M. Okamura, and E. W. Hawkes, "Obstacle-Aided Navigation of a Soft Growing Robot," in 2018 IEEE International Conference on Robotics and Automation (ICRA), Brisbane, QLD, May 2018, pp. 1-8, doi: 10.1109/ICRA.2018.8460777.

[30] J. D. Greer, T. K. Morimoto, A. M. Okamura, and E. W. Hawkes, "A Soft, Steerable Continuum Robot That Grows via Tip Extension," Soft Robot., vol. 6, no. 1, pp. 95-108, Feb. 2019, doi: 10.1089/soro.2018.0034.

[31] J. D. Greer, T. K. Morimoto, A. M. Okamura, and E. W. Hawkes, "Series pneumatic artificial muscles (sPAMs) and application to a soft continuum robot," May 2017, pp. 5503-5510, doi: 10.1109/ICRA.2017.7989648.

[32] A. Ataka, T. Abrar, F. Putzu, H. Godaba, and K. Althoefer, "Model-Based Pose Control of Inflatable Eversion Robot With Variable Stiffness," IEEE Robot. Autom. Lett., vol. 5, no. 2, pp. 3398-3405, Apr. 2020, doi: 10.1109/LRA.2020.2976326.

[33] A. Ataka, T. Abrar, F. Putzu, H. Godaba, and K. Althoefer, "Observer-based Control of Inflatable Robot with Variable Stiffness," p. 7.

[34] N. Kellaris, V. Gopaluni Venkata, G. M. Smith, S. K. Mitchell, and C. Keplinger, "Peano-HASEL actuators: Muscle-mimetic, electrohydraulic transducers that linearly contract on activation," Sci. Robot., vol. 3, no. 14, p. eaar3276, Jan. 2018, doi: 10.1126/scirobotics.aar3276.

[35] H. Godaba, F. Putzu, T. Abrar, J. Konstantinova, and K. Althoefer, "Payload Capabilities and Operational Limits of Eversion Robots," in Towards Autonomous Robotic Systems, vol. 11650, K. Althoefer, J. Konstantinova, and K. Zhang, Eds. Cham: Springer International Publishing, 2019, pp. 383-394. 